\title{
A Spherical-magnet End-effector for Robotic Magnetic Manipulation
}

\author{
Samuel E. Wright, Arthur W. Mahoney, Katie M. Popek, and Jake J. Abbott
}

\begin{abstract}
A variety of magnetic devices can be manipulated remotely using a single permanent "actuator" magnet positioned in space by a robotic manipulator. This paper presents the spherical-actuator-magnet manipulator (SAMM), which is designed to replace or augment the singularity-prone spherical wrist used by prior permanent-magnet manipulation systems. The SAMM uses three omniwheels to enable holonomic control of a spherical magnet's heading and enable the magnet's instantaneous axis-of-rotation to be set arbitrarily. The SAMM performs closed-loop control of its dipole using field measurements obtained from Hall-effect sensors. We describe the operation and construction of the SAMM, develop and characterize a controller for the SAMM's spherical magnet, and demonstrate remote actuation of an untethered magnetic device in a lumen.
\end{abstract}

\section{INTRODUCTION}

This paper presents a mechatronic device that we refer to as the spherical-actuator-magnet manipulator (SAMM), which enables holonomic singularity-free control of a spherical permanent magnet's orientation, to act as the actuator magnet in a magnetic manipulation system (Fig. 1). The SAMM is intended to be used as an end-effector mounted to the tool frame of a robotic manipulator, so as to remove kinematic limitations encountered in prior permanent-magnet manipulation systems. The SAMM's spherical magnet is driven by three omniwheels that contact the magnet's surface. An omniwheel is a common mechanism that incorporates small rollers that permit controlled rotation about the omniwheel's rotation axis and free rotation about the two orthogonal axes. Designing the three omniwheel rotation axes to be linearly independent enables any instantaneous magnet rotation axis to be achieved. By making the magnet's axis-of-rotation continuously variable, irrespective of the robotic manipulator used to position the SAMM, the kinematic singularities of the robotic manipulator can be avoided, and the robotic manipulator is free to position the actuator magnet optimally for manipulation. The SAMM will also enable robotic manipulators with less than six degrees of freedom (6-DOF) to be considered for use in magnetic manipulation. The SAMM has the potential to be used for the remote actuation of a variety of magnetic devices that have been previously developed for minimally invasive medicine, including both untethered magnetic devices (UMDs) [1][10], and tethered magentic devices [11], [12].

This work was supported in part by the National Science Foundation under Grant No. 0952718.

S. E. Wright and J. J. Abbott are with the Department of Mechanical Engineering, and K. M. Popek is with the School of Computing, University of Utah, USA. A. W. Mahoney is with the Department of Mechanical Engineering, Vanderbilt University, USA. jake. abbott @utah. edu

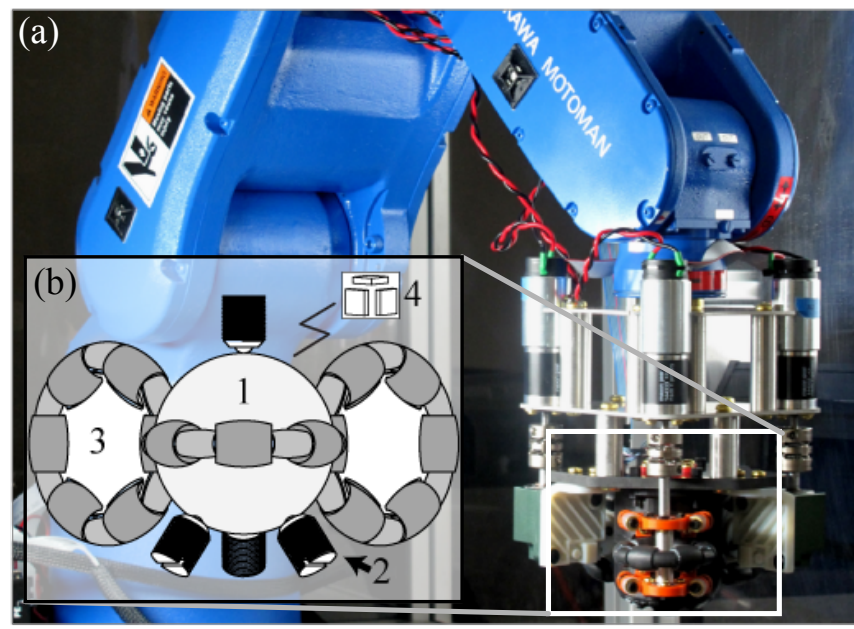

Fig. 1. (a) The spherical-actuator-magnet manipulator (SAMM) prototype as the end-effector of a robotic manipulator. (b) A concept diagram illustrating the spherical magnet (1), which is prevented from translating by four constraints (2) that create a rolling form closure. Three omniwheels (3) whose axes of rotation span $\mathbb{R}^{3}$ contact the magnet and cause it to rotate as desired. Magnetic-field sensors (4) measure the magnet's dipole moment to be used for closed-loop control of the dipole's orientation.

Two prior works in our lab motivated the development of the SAMM. The ability to control a UMD in a luman using a single rotating permanent magnet as the actuator magnet was described in [10], in a task reminiscent of active capsule endoscopy in the intestines. The results of [10] enable the actuator magnet to be placed in any position relative to the UMD, provided a specific position-dependent actuator-magnet rotation axis is established. In the experimental results of [10], the actuator magnet was rotated by a single DC motor that was rigidly mounted to the tool frame of an industrial 6-DOF robotic manipulator. In that setup, the rotation axis of the actuator magnet was fixed with respect to the tool frame of the robotic manipulator. Such a setup is capable of placing the actuator magnet with the correct rotation axis to guide a UMD through relatively simple trajectories. However, when tasked with navigating a UMD through tortuous paths (e.g., the small intestines), the physical constraints of the robotic manipulator (i.e., joint limits and singularities) limit how the UMD can be actuated, and limit the workspace.

The effects of manipulator limitations on UMD actuation were also observed and were characterized in [13], where a single, nonrotating permanent magnet was used to levitate a semibuoyant magnetic capsule with 5-DOF (3-DOF position and 2-DOF heading) control in a task reminiscent of cap- 
sule endoscopy in the stomach. Kinematic singularities and workspace limitations were identified as the primary limiting factors to dexterous manipulation. To mitigate the effect of singularities, the authors introduced a control method that sacrificed control authority over the capsule's heading in order to maintain 3-DOF control over the capsule's position when the manipulator nears a kinematic singularity.

The SAMM, with its singularity-free orientation control of its spherical magnet, can be used to solve the problems found in both of the projects described above.

There are several reasons for actuating a magnet of spherical geometry. Firstly, being of constant radius, it is simple to maintain form-closure regardless of the magnet's orientation, allowing it to be easily incorporated into a physical device. Secondly, a spherical magnet makes the best use of available space in the sense that it fully utilizes the volume of its bounding sphere. Thirdly, the field of a spherical magnet is theoretically perfectly fit by the analytical point-dipole model. Finally, a spherical body has no principle directions of inertia, giving it isotropic dynamic properties.

Our SAMM design was inspired by prior "ballbot" systems, in which a robot balances itself atop a sphere (e.g., a bowling ball) [14], [15]. With ballbots, only the instantaneous angular velocity of the ball is important for control, and the ball's orientation is not measured [16] (i.e., there is no preferred "north pole" of a bowling ball). However, for remote magnetic manipulation, knowledge of the magnet's dipole-heading is critical since it determines the magnetic field applied to the actuated magnetic device and how the device is controlled. Therefore, the SAMM includes a magnetic-field sensor system to estimate the sphericalmagnet's dipole heading (we say "heading" since the dipole's magnitude is constant and known).

\section{Velocity Kinematics And InVERse Kinematics}

We follow a convention where scalars are denoted by lower-case standard font (e.g., c), vectors by lower-case bold font (e.g., x), and matrices by capital bold font (e.g., M). The ^symbol denotes a unit-length vector (e.g., $\hat{\mathbf{x}}$ ).

For some desired angular velocity $\omega_{\mathbf{m}} \in \mathbb{R}^{3}$ of the spherical magnet, the necessary omniwheel rotation speeds must be determined. Let the unit-length vectors $\hat{\mathbf{d}}_{1}, \hat{\mathbf{d}}_{2}$, and $\hat{\mathbf{d}}_{3}$ point from the magnet's center to the contact point where each of the three omniwheels touches the magnet (Fig. 2). We assume that the omniwheel axes $\hat{\mathbf{a}}_{1}, \hat{\mathbf{a}}_{2}$, and $\hat{\mathbf{a}}_{3}$ are perpendicular to $\hat{\mathbf{d}}_{1}, \hat{\mathbf{d}}_{2}$, and $\hat{\mathbf{d}}_{3}$, respectively, and that there is no slip between the omniwheels and the magnet. Given a magnet angular velocity $\omega_{\mathbf{m}}$, the surface velocity of the magnet at the $i^{\text {th }}$ omniwheel-magnet contact point is

$$
\mathbf{u}_{i}=r_{m} \boldsymbol{\omega}_{\mathbf{m}} \times \hat{\mathbf{d}}_{i},
$$

where $r_{m}$ is the radius of the magnet.

The components of $\mathbf{u}_{1}, \mathbf{u}_{2}$, and $\mathbf{u}_{3}$ parallel to the respective omniwheel axes are transferred directly into rotation of the omniwheel rollers, and cause no rotation of the omniwheels themselves. All other components of $\mathbf{u}_{1}, \mathbf{u}_{2}$, and $\mathbf{u}_{3}$ cause each omniwheel to rotate with scalar rotation speeds
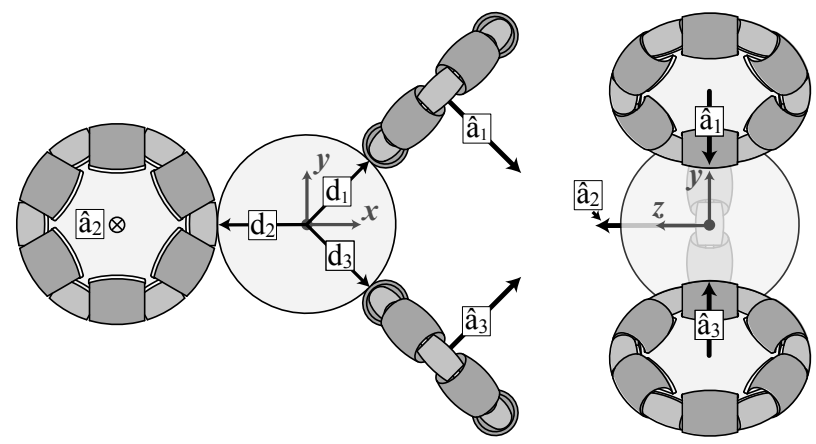

Fig. 2. Two orthogonal views of the SAMM are shown. The vectors $\hat{\mathbf{a}}_{1}$, $\hat{\mathbf{a}}_{2}$, and $\hat{\mathbf{a}}_{3}$ are the omniwheel rotation axes, and $\hat{\mathbf{d}}_{1}, \hat{\mathbf{d}}_{2}$, and $\hat{\mathbf{d}}_{3}$ point from the magnet center to the corresponding omniwheel contact point. The depicted coordinate system is used throughout this paper.

$\omega_{\mathrm{a} 1}, \omega_{\mathrm{a} 2}$, and $\omega_{\mathrm{a} 3}$, respectively. The component direction of $\mathbf{u}_{i}$ that causes the $i^{\text {th }}$ omniwheel to rotate about its axis is

$$
\hat{\mathbf{q}}_{i}=\hat{\mathbf{d}}_{i} \times \hat{\mathbf{a}}_{i} \text {. }
$$

Under the assumption of no-slip, the projection of $\mathbf{u}_{1}, \mathbf{u}_{2}$, and $\mathbf{u}_{3}$ onto the directions $\hat{\mathbf{q}}_{1}, \hat{\mathbf{q}}_{2}$, and $\hat{\mathbf{q}}_{3}$, respectively, must be mapped to the scalar rotation speeds of each omniwheel by the reciprocal of the omniwheels' radii (denoted by $r_{w}$, as we assume identical omniwheels) as

$$
\omega_{\mathrm{a} i}=\frac{1}{r_{w}} \hat{\mathbf{q}}_{i}^{\top} \mathbf{u}_{i}=\frac{r_{m}}{r_{w}} \hat{\mathbf{a}}_{i}^{\top}\left\{\hat{\mathbf{d}}_{i}\right\}^{2} \boldsymbol{\omega}_{\mathbf{m}},
$$

where $\left\{\hat{\mathbf{d}}_{\mathbf{i}}\right\} \in \operatorname{so}(3)$ is the skew-symmetric matrix form of the cross-product operation.

All three omniwheel rotation speeds can be packed into the vector $\omega_{\mathbf{a}}$ and related to the spherical magnet angular velocity $\omega_{m}$, in matrix form, as

$$
\boldsymbol{\omega}_{\mathbf{a}}=\left[\begin{array}{c}
\omega_{\mathrm{a} 1} \\
\omega_{\mathrm{a} 2} \\
\omega_{\mathrm{a} 3}
\end{array}\right]=\frac{r_{m}}{r_{w}}\left[\begin{array}{l}
\hat{\mathbf{a}}_{1}^{\top}\left\{\hat{\mathbf{d}}_{1}\right\}^{2} \\
\hat{\mathbf{a}}_{2}^{\top}\left\{\hat{\mathbf{d}}_{2}\right\}^{2} \\
\hat{\mathbf{a}}_{3}^{\top}\left\{\hat{\mathbf{d}}_{3}\right\}^{2}
\end{array}\right] \boldsymbol{\omega}_{\mathbf{m}} .
$$

Due to the assumption that $\hat{\mathbf{a}}_{\mathbf{i}}$ is perpendicular to $\hat{\mathbf{d}}_{\mathbf{i}}$, (4) can be simplified to

$$
\boldsymbol{\omega}_{\mathbf{a}}=\frac{r_{m}}{r_{w}}\left[\begin{array}{c}
-\hat{\mathbf{a}}_{1}^{\top} \\
-\hat{\mathbf{a}}_{2}^{\top} \\
-\hat{\mathbf{a}}_{3}^{\top}
\end{array}\right] \boldsymbol{\omega}_{\mathbf{m}}=\eta \mathbf{A}^{\top} \boldsymbol{\omega}_{\mathbf{m}},
$$

where $\eta=-r_{m} / r_{w}$ is the gear ratio from the omniwheels to the sphere (with the negative sign indicating the change in rotation direction from the omniwheels to the magnet), and where $\mathbf{A}=\left[\begin{array}{lll}\hat{\mathbf{a}}_{1} & \hat{\mathbf{a}}_{2} & \hat{\mathbf{a}}_{3}\end{array}\right]$.

The omniwheel axes and positioning must be designed such that the matrix $\mathbf{A}$ has full rank, otherwise there will exist a direction of $\omega_{m}$ that cannot be achieved with any selection of omniwheel rotation speeds. Although linear independence of the columns of $\mathbf{A}$ is a sufficient condition mathematically, in practice the columns should be designed to be as close to mutually orthogonal as possible. Otherwise, some desired $\omega_{\mathbf{m}}$ will require an unnecessarily, and possibly 
unachievably, fast omniwheel rotation speed. We designed our system so that $\hat{\mathbf{a}}_{1}, \hat{\mathbf{a}}_{2}$, and $\hat{\mathbf{a}}_{3}$ are mutually perpendicular.

\section{Detecting the Magnet's Dipole Moment}

The dipole moment of the spherical magnetic, denoted by the vector $\mathbf{m} \in \mathbb{R}^{3}$, is the vector from the south to north poles of the magnet. The dipole moment $\mathbf{m}$ of the SAMM's magnet can be determined by measuring the magnetic field $\mathbf{h}$ that it generates in space. One approach to measuring the magnetic field uses Hall-effect sensors. Hall-effect sensors measure the component of the field in the direction normal to (i.e., passing through) the sensor's face. We assume the general case of $n$ Hall-effect sensors. Let each sensor be positioned in space such that the vectors $\mathbf{p}_{1}$ through $\mathbf{p}_{n}$, in units of meters, measure each sensor's position relative to the spherical magnet's center, and let $\hat{\mathbf{v}}_{1}$ through $\hat{\mathbf{v}}_{n}$ be unit-magnitude vectors that describe the directions that are sensed by each sensor. Let the magnetic field at each sensor position be denoted by $\mathbf{h}_{1}$ through $\mathbf{h}_{n}$, in units $\mathrm{A} \cdot \mathrm{m}^{-1}$. The measured component of the field produced by the $i^{\text {th }}$ sensor is denoted with the scalar $s_{i}$ and is given by

$$
s_{i}=\hat{\mathbf{v}}_{i}^{\top} \mathbf{h}_{i}
$$

The field $\mathbf{h}_{i}$ of a spherical magnet, at each sensor position $\mathbf{p}_{i}$, can be accurately predicted with the point-dipole model

$$
\mathbf{h}_{i}=\frac{1}{4 \pi\left\|\mathbf{p}_{i}\right\|^{3}}\left(3 \hat{\mathbf{p}}_{i} \hat{\mathbf{p}}_{i}^{\top}-\mathbf{I}_{\mathbf{3}}\right) \mathbf{m}=\mathbf{H}_{i} \mathbf{m},
$$

where $\mathbf{I}_{\mathbf{3}} \in \mathbb{R}^{3 \times 3}$ is the identity matrix.

Substituting (7) into (6) relates the magnet's dipole moment $\mathbf{m}$ to each of the $n$ sensor measurements, which can be aggregated into the matrix equation

$$
\mathbf{s}=\left[\begin{array}{c}
s_{1} \\
\vdots \\
s_{n}
\end{array}\right]=\left[\begin{array}{c}
\hat{\mathbf{v}}_{1}^{\top} \mathbf{H}_{1} \\
\vdots \\
\hat{\mathbf{v}}_{n}^{\top} \mathbf{H}_{n}
\end{array}\right] \mathbf{m}=\mathbf{S m}
$$

The $n \times 3$ constant matrix $\mathbf{S}$ encapsulates the complete geometric description of the sensor arrangement, as it pertains to estimating $\mathbf{~ m}$. If the matrix $\mathbf{S}$ has full column rank, then a solution for the dipole moment $\mathbf{m}$ can be found as

$$
\mathbf{m}=\mathbf{S}^{\dagger} \mathbf{s}
$$

where $\mathbf{S}^{\dagger}$ is the Moore-Penrose pseudoinverse of $\mathbf{S}$, which is constant and can be calculated off-line. The matrix $\mathbf{S}$ should be made to have full column rank by using at least three Hall-effect sensors and appropriately selecting the positions $\left(\mathbf{p}_{i}\right)$ and directions $\left(\hat{\mathbf{v}}_{i}\right)$ of each sensor. When $n>3$, (9) provides the best estimate of $\mathbf{m}$ in a least-squares sense. Although (9) can provide an instantaneous reading of the magnet's measured dipole, in practice a Kalman filter can be employed to incorporate the manipulator's dynamics with the sensor readings to reduce noise in the system.

\section{DESIGN}

Our prototype SAMM is shown in Fig. 3. The magnet is a 50.8-mm-diameter, Grade-N42, spherical permanent magnet with a dipole strength of $71.6 \mathrm{~A} \cdot \mathrm{m}^{2}$. The form-closure constraints that allow only rotation of the spherical magnet are implemented with four ball-roller-tipped non-magnetic set-screws. Housed inside the tip of each set-screw is a freely rotating $5.56-\mathrm{mm}$ ball that is supported by $1.50-\mathrm{mm}$ sub-rollers. The body of the set-screws are nylon and the ball-tip and subrollers are ceramic. The SAMM housing resembles a cylindrical structure with a hemisphere at one end where three form-closure constraints are mounted. The housing is constructed out of nonconductive ABS plastic to mitigate eddy-current damping. The omniwheels contact the magnet through windows in the cylindrical body. Their counter-opposed configuration allows the normal forces from one omniwheel to be transmitted to the other omniwheels, which mutually increases their traction. The omniwheel axes are $\hat{\mathbf{a}}_{\mathbf{1}}=[\sqrt{2} / 2, \sqrt{2} / 2,0]^{\top}, \hat{\mathbf{a}}_{\mathbf{2}}=[0,0,-1]^{\top}$, and $\hat{\mathbf{a}}_{\mathbf{3}}=$ $[\sqrt{2} / 2,-\sqrt{2} / 2,0]^{\top}$ in the coordinate system of Fig. 2 .

In the configuration shown in Fig. 3, the SAMM can be positioned so that the device's "bottom" (the hemispherical side where three of the four set-screws are located), which is streamlined and free of moving parts, is presented to the manipulation workspace, reducing the risk of damage to the moving SAMM components and enabling the spherical actuator magnet to be positioned close to the remote magnetic device being manipulated.

The omniwheels employed on the SAMM are miniaturized, nonmagnetic, continuous-contact omniwheels, modified from the designs in [14], [17]. The omniwheels contact the magnet with rollers that generate high friction in the driving direction while allowing the magnet to passively roll orthogonal to the drive direction with minimal friction. The major diameter of each assembled omniwheel is $58.2 \mathrm{~mm}$.

The omniwheels are driven by three Maxon RE-max 29 gearmotors, which have a 24:1 gear ratio and 512 CPT encoders, mounted in a parallel arrangement (Fig. 3). The torques applied to omniwheel axes $\hat{\mathbf{a}}_{1}$ and $\hat{\mathbf{a}}_{3}$ are redirected via $90^{\circ}$ gearboxes; the torque applied to omniwheel axis $\hat{\mathbf{a}}_{2}$ is transmitted via direct-drive. The $90^{\circ}$ gearboxes consist of nylon gears mounted to aluminum shafts supported by dual acetal ball bearings inside an aluminum case, making the $90^{\circ}$ gearboxes entirely nonmagnetic. The gearmotors mount to the gearboxes (and to the omniwheel drive-shafts in the case of axis $\hat{a}_{2}$ ) by aluminum helical couplings.

The prototype SAMM employs two approaches to buildin compliance that keeps the omniwheels in contact with the magnet despite irregularities in their construction: 1-DOF rotary motion is employed on the $1^{\text {st }}$ and $3^{\text {rd }}$ omniwheels, and approximate straight-line motion of the $2^{\text {nd }}$ omniwheel is achieved with two pillow blocks. In the rotary case, both rotary axes lie parallel to their respective omniwheel axes (i.e., $\hat{\mathbf{a}}_{1}$ and $\hat{\mathbf{a}}_{3}$ ). The $90^{\circ}$ gearboxes make the rotary axis perpendicular to the respective motor axis, which decouples the compliance from the motor torque transmission. If the 


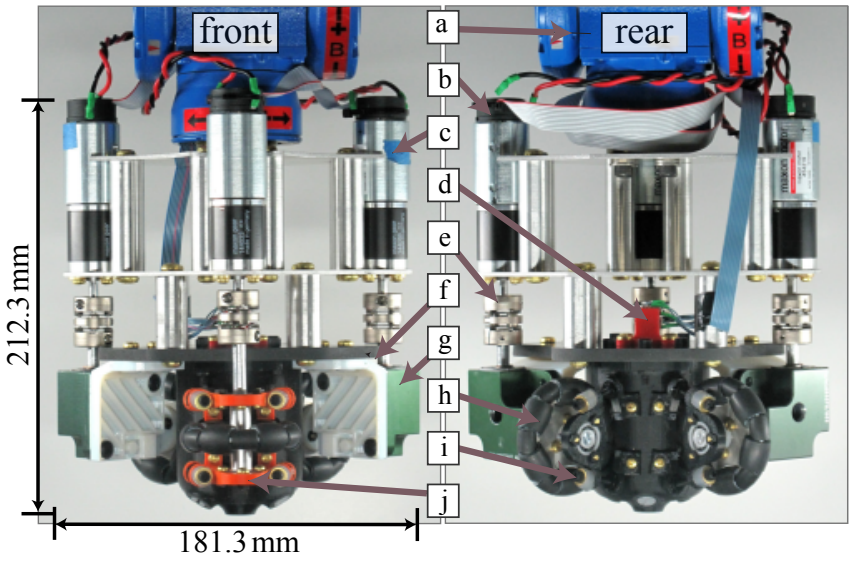

Fig. 3. Prototype SAMM shown mounted to the robotic manipulator (a). Encoders (b) measure the gearmotors' (c) position. The cluster of Hall-effect sensors (d) measures the spherical magnet's dipole. Power is transmitted through helical shaft couplings (e) to omniwheel axles or $90^{\circ}$ gearboxes $(\mathrm{g})$, which pivot for omniwheel compliance (f). Omniwheels (h) are tensioned to the spherical magnet through adjustable spring-tensioned pillow blocks (i), whose tension can be manually tuned through adjustment screws (j). As viewed from the front, the SAMM is $142.1 \mathrm{~mm}$ deep.

direction of compliance were not decoupled from the motortorque transmission direction, then both omniwheel irregularities and torque transmission would cause the tensioning mechanisms to move in the direction of compliance, resulting in undesirable friction or traction loss.

Tension is applied to the $1^{\text {st }}$ and $3^{\text {rd }}$ omniwheel assemblies by adjustable spring-tensioned pillow blocks. The pillow blocks are constructed of 3D-printed ABS plastic with cutouts revealing serpentine shaped springs. The serpentine spring is reinforced with a silicone compression spring (visible in Fig. 3) whose tension is adjusted via an adjustment screw. On the $2^{\text {nd }}$ omniwheel, approximate straight-line motion is formed utilizing two of the same adjustable springtensioned pillow blocks as on the $1^{\text {st }}$ and $3^{\text {rd }}$ axes, to tension the omniwheel directly onto the magnet in the direction $\hat{\mathbf{d}}_{2}$. Although the motion is not strictly constrained to $\hat{\mathbf{d}}_{2}$, we have found that the deviation is small and results in insignificant changes to the $\mathbf{A}$ matrix.

A sensor cluster, consisting of six 1-DOF Allegro A1302 Hall-effect sensors arranged on the surface of a $7.5 \mathrm{~mm}$ cube, is mounted to the housing as shown in Figs. 3(d) and 4. In addition to being a space free from moving parts, this location ensures that magnetic-field disturbances in the workspace below the SAMM (e.g., from the magnet of a device being manipulated by the SAMM) have a minimal impact on the estimation of the actuating magnet's dipole heading. The gearmotors are the nearest magnetic component to the sensor cluster, but they are positioned sufficiently far away to make their contribution to the measured magnetic field negligible. The sensors are chosen with a sensitivity of $13 \mathrm{mV} / \mathrm{mT}$, which utilizes their full output-voltage range without saturation.

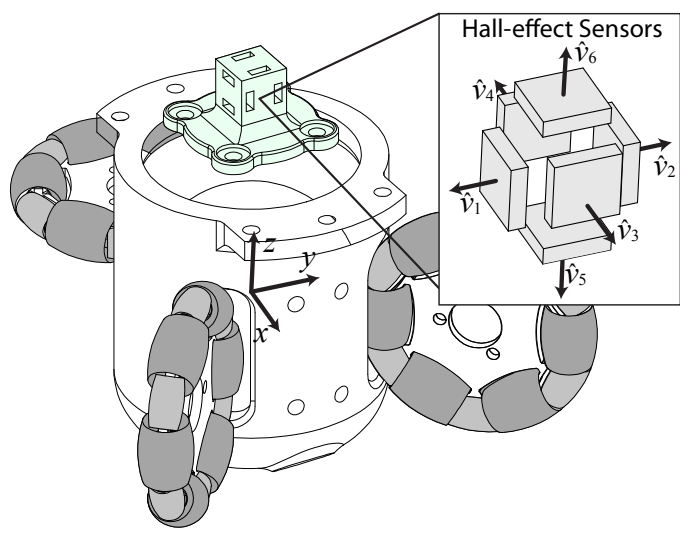

Fig. 4. The pose of the spherical magnet's dipole moment is estimated with a cluster of six 1-DOF Hall-effect sensor arranged on the surface of a 7.5 $\mathrm{mm}$ cube. The sensor assembly is mounted to the SAMM housing where there are no moving parts and magnetic-field disturbances are minimal.

\section{Control}

The SAMM has two modes of operation: pointing and rotating. The pointing-mode controller is simply a PID controller to regulate the dipole heading to some desired heading, to be used for the generation of quasistatic fields. For brevity, we will not discuss the pointing-mode controller in greater detail here. The purpose of the rotating-mode controller is to generate continuous rotation of the actuatormagnet dipole with some desired angular velocity $\kappa$, with the dipole orthogonal to $\kappa$, without any concern for the phase of the dipole within the cycle. Examples where the rotating mode would be useful include tasks where a rotating field is fundamental to the actuation strategy (e.g., [4]-[10]).

The Maxon-Motor controllers take as input a desired motor angular velocity $\sigma$, but since they act on that input in a model-based open-loop fashion (in our chosen implementation), there is no guarantee that the motor's angular velocity will achieve the desired, necessitating the inclusion of our own custom controller to set $\boldsymbol{\sigma}$.

The rotating-mode controller employs two subcontrollers: a PI-plus-feed-forward angular velocity subcontroller that rotates $\hat{\mathbf{m}}$ about a desired angular-velocity vector $\boldsymbol{\kappa}$ with control effort given by $\sigma_{\|}$, which is parallel to $\kappa$, and a PD heading subcontroller to drive $\hat{\mathbf{m}}$ to the plane orthogonal to $\kappa$ with control effort given by $\sigma_{\perp}$. These controllers are independent from each other, as they always actuate orthogonal to one another. The two orthogonal control laws are combined to form the total output

$$
\sigma=\sigma_{\|}+\sigma_{\perp} .
$$

To determine control effort in the direction parallel to $\kappa$, the angular-velocity error $\mathbf{e}_{\|}$must first be computed:

$$
\mathbf{e}_{\|}=\kappa-\left(\omega_{\mathbf{m}} \cdot \hat{\boldsymbol{\kappa}}\right) \hat{\boldsymbol{\kappa}} .
$$

Control effort in the direction parallel to $\kappa$, which governs angular velocity, can then be computed at time-sample $j$ as

$$
\boldsymbol{\sigma}_{\|, j}=\boldsymbol{\kappa}_{j}+k_{p \|} \mathbf{e}_{\|, j}+k_{i \|}\left(\mathbf{q}_{\epsilon}\left(\mathbf{e}_{\|, j}\right) \cdot \hat{\boldsymbol{\kappa}}_{j}\right) \hat{\boldsymbol{\kappa}}_{j},
$$


where the " $\kappa$ " term represents a feed-forward component, and the behavior of the capped integration function $\mathbf{q}_{\epsilon}$ is defined as

$$
\mathbf{q}_{\epsilon}\left(\mathbf{z}_{j}\right)=\left\{\begin{array}{rl}
\mathbf{q}_{\epsilon}\left(\mathbf{z}_{j-1}\right)+\delta \mathbf{z}_{j}: & \left\|\mathbf{q}_{\epsilon}\left(\mathbf{z}_{j-1}\right)+\delta \mathbf{z}_{j}\right\| \leq \epsilon \\
\mathbf{q}_{\epsilon}\left(\mathbf{z}_{j-1}\right): & \left\|\mathbf{q}_{\epsilon}\left(\mathbf{z}_{j-1}\right)+\delta \mathbf{z}_{j}\right\|>\epsilon
\end{array},\right.
$$

where $\delta$ is the time step. The term $\mathbf{q}_{\epsilon}$ is projected onto the rotation axis parallel to $\kappa$ to alleviate the effects of integrator wind-up in what is currently the wrong direction.

To determine control effort orthogonal to $\kappa$, we compute a rotation-plane restoration vector as

$$
\mathbf{e}_{\perp}=\hat{\mathbf{m}} \times\left(\mathbf{I}_{\mathbf{3}}-\hat{\boldsymbol{\kappa}} \hat{\boldsymbol{\kappa}}^{\top}\right) \hat{\mathbf{m}},
$$

which approximates the angular error between the dipole and the desired plane at small angles. Control effort can then be computed at time-sample $j$ as

$$
\begin{aligned}
\boldsymbol{\sigma}_{\perp, j} & =\xi_{j}\left(k_{p \perp}\left\|\mathbf{e}_{\perp, j}\right\| \xi_{j}\right. \\
& \left.+k_{d \perp} \operatorname{sat}_{v}\left(\frac{\left\|\mathbf{e}_{\perp, j}\right\| \xi_{j}-\left\|\mathbf{e}_{\perp, j-1}\right\| \xi_{j-1}}{\delta}\right)\right) \hat{\mathbf{e}}_{\perp, j}
\end{aligned}
$$

where $\xi_{j}=\operatorname{sgn}\left(\boldsymbol{\omega}_{\mathbf{m}, j} \cdot \hat{\mathbf{m}}_{j}\right)$ is a signum function that is used to correctly account for signed rotation-plane error (i.e., determine if $\hat{\mathbf{m}}$ is above or below the desired plane), the gains $k_{p \perp}$ and $k_{d \perp}$ are proportional and derivative gains, and the saturation function $\mathrm{sat}_{v}$ is defined as

$$
\operatorname{sat}_{v}(\mathbf{z})=\left\{\begin{aligned}
\mathbf{z}: & \|\mathbf{z}\| \leq v \\
\hat{\mathbf{z}} v: & \|\mathbf{z}\|>v
\end{aligned}\right.
$$

The gains $k_{p \perp}$ and $k_{d \perp}$ first act upon the magnitude of the rotation-plane restoration vector $\left\|\mathbf{e}_{\perp}\right\|$, and are then applied in the direction of the rotation-plane restoration vector $\hat{\mathbf{e}}_{\perp}$. The inclusion of the signum function is necessary because the direction of $\mathbf{e}_{\perp}$, computed with (14), flips when the dipole crosses through the desired plane.

\section{EXPERIMENTATION}

Experiments were performed with the SAMM mounted to a 6-DOF Yaskawa Motoman MH5 robotic arm (Fig. 1). The SAMM weighs $1.4 \mathrm{~kg}$, which is below the arm's reported maximum payload weight of $5 \mathrm{~kg}$. The control system was implemented in $\mathrm{C}++$, with a Sensoray 626 PCI DAQ card, and operates at $1000 \mathrm{~Hz}$.

We used the Ziegler-Nichols tuning method to tune the PD gains of the "orthogonal" heading subcontroller as $k_{p \perp}=$ $17.2 \mathrm{rad} / \mathrm{s}$ and $k_{d \perp}=1.40 \mathrm{rad}$. The resulting PD controller had a settling time of approximately $t_{s}=1.5 \mathrm{~s}$, which is equivalent to a time constant of approximately $\tau=0.3 \mathrm{~s}$. The PI gains of the angular-velocity subcontroller were tuned to so that the angular-velocity and heading (i.e., orthogonal) subcontrollers converge to their respective set-points with similar time constants. This yields the gains of $k_{p \|}=0.187$, and $k_{i \|}=0.460 \mathrm{~s}^{-1}$. The derivative terms were clipped using $\nu=100$, which allows the derivative to function unimpeded during the majority of its operation, only clipping the most extreme derivative spikes. The integral terms were capped at $\epsilon=1$. This allows the integral term to generally operate unimpaired, and was rarely observed to grow large, but prevents excessive wind-up in extreme cases.

To demonstrate the function of the rotating-mode controller, we chose a desired angular velocity $\kappa=\pi$. $[\sqrt{2} / 2,0, \sqrt{2} / 2]^{\top} \mathrm{rad} / \mathrm{s}$, since it is not aligned with any of the omniwheel axes, and it has a magnitude that is similar to what has been typically used when propelling screw-type capsule endoscopes. We initialized the dipole at $[0,0,1]^{\top}$, which has a relatively large error with respect to the desired rotation plane. The trajectory of the dipole can be viewed from multiple angles in Fig.5(a). In this experiment, the dipole heading is pulled into the desired rotation plane within its first revolution about $\kappa$. The rotation-plane error plot illustrates the rotation-plane heading subcontroller resulting in an RMS error of 0.0347 rad for the final $50 \%$ of the test, keeping the dipole within approximately $\pm 5^{\circ}$ of the desired plane. The angular-velocity error plot illustrates the PI-plus-feedforward angular-velocity subcontroller resulting in an RMS error of $0.070 \mathrm{rad} / \mathrm{s}$ for the final $50 \%$ of the test. We can also observe two instances in the angularvelocity error where the error suddenly increased (possibly due to omniwheel slip or a high-friction region on the rolling constraints), which the controller rejects.

In [10], we performed experiments where a spherical UMD was actuated down a lumen using a rotating field, generated by a cylindrical permanent magnet, and the UMD position $\mathbf{p}$ was continuously measured by a stereo-camera system. The actuator magnet was rigidly attached to the shaft of a DC motor, which was maneuvered in space by the same manipulator shown in Fig. 1 to ensure that the magnet's rotation axis $\hat{\kappa}$ is set according to

$$
\hat{\boldsymbol{\kappa}}=\widehat{\mathbf{H}(\mathbf{p}) \hat{\boldsymbol{\kappa}}_{\mathbf{h}}}
$$

for all time, where $\hat{\boldsymbol{\kappa}}_{\mathbf{h}}$ is the magnetic-field rotation axis that causes the spherical UMD to rotate down the lumen [10], and $\mathbf{H}(\mathbf{p})=3 \hat{\mathbf{p}} \hat{\mathbf{p}}^{\top}-\mathbf{I}_{3}$ is from the point-dipole model (7).

In one experiment, the spherical UMD was rolled down the lumen while the position of the actuator magnet was kept stationary [Fig. 5(b)], which requires the actuator-magnet's rotation axis, and thus the robot manipulator's wrist, to turn almost $180^{\circ}$. For comparison, Fig. 5(c) shows the same experiment using the SAMM end-effector, but in this case, the manipulator remains completely stationary as shown.

In another example, the UMD was rolled down the lumen while the position of the actuator magnet follows a steptrajectory independent of the UMD's position [Fig. 5(d)]. In this experiment, the necessary actuator-magnet rotation axis (17) forces the robot manipulator's wrist to contort dramatically (nearly violating joint limits at $t=77 \mathrm{~s}$ ). For comparison, Fig. 5(e) shows a similar experiment performed with the SAMM end-effector. In this case, the manipulator's wrist remains nearly stationary throughout the trajectory, only changing slightly to keep the SAMM in a constant orientation. In both experiments, the SAMM dramatically reduces the manipulator motion required to perform the 

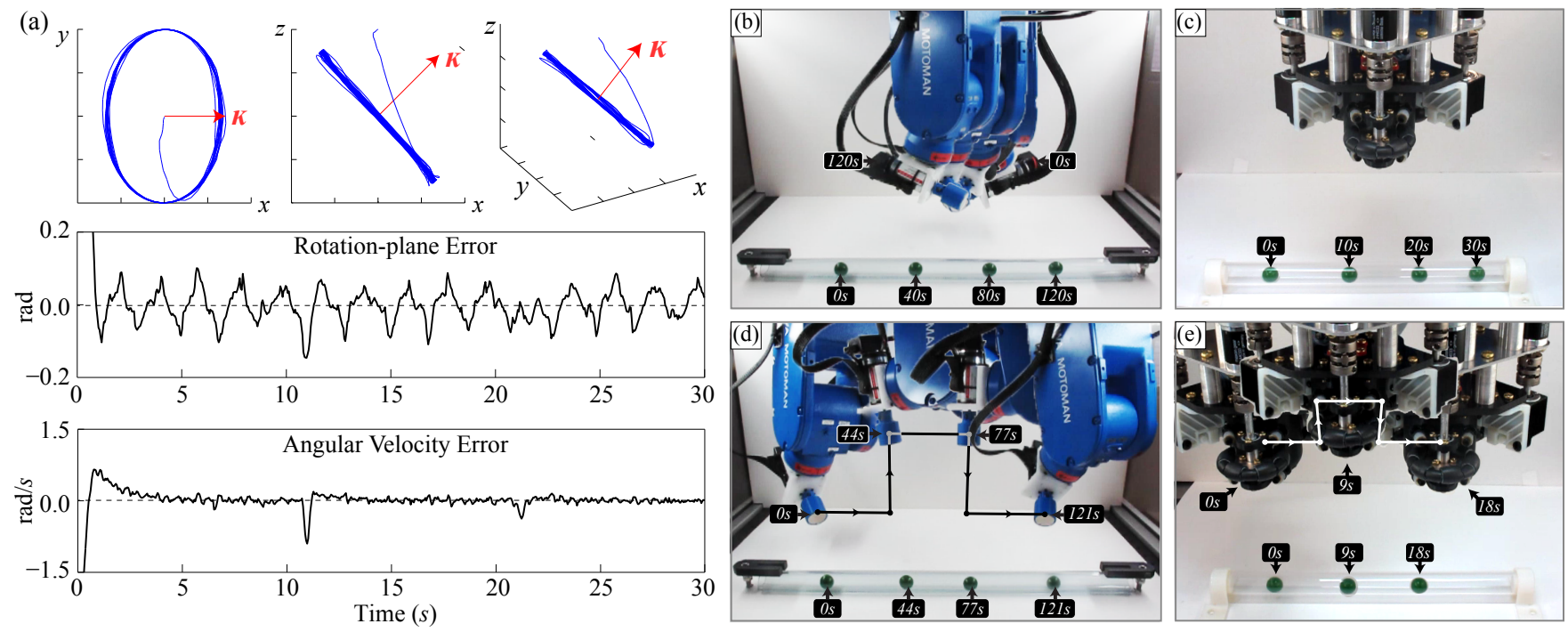

Fig. 5. (a) Recorded dipole heading, rotation-plane error, and angular-velocity error, with the dipole starting from rest at $[0,0,1]^{\top}$, and commanded to rotate with angular velocity $\kappa=\pi \cdot[\sqrt{2} / 2,0, \sqrt{2} / 2]^{\top} \mathrm{rad} / \mathrm{s}$ expressed in the coordinate system of Fig. 2. (b) In prior work [10], a spherical UMD was rolled down a lumen using a rotating field generated by a rotating permanent-magnet actuator, whose position was held stationary in space while its rotation axis is set according to (17). This maneuver required the robot manipulator's wrist to turn almost $180^{\circ}$. (c) For comparison, we used the SAMM to perform the same maneuver, which required no motion of the robot manipulator. (d) In another example, the UMD is rolled down a lumen while the rotating permanent-magnet actuator's position follows a square step-trajectory independent of the UMD's position, which required the manipulator's wrist to contort dramatically to satisfy (17). (e) For comparison, we performed a similar experiment using the SAMM. In this case, the manipulator's wrist only moves to keep the SAMM in a constant orientation.

maneuvers. We note that both experiments were possible with the SAMM held in a constant orientation, demonstrating that a much simpler robot manipulator (e.g., a Cartesian robot) could have been used to accomplish the same results.

\section{CONCLUSION}

We have presented a singularity-free, holonomic mechatronic device, the spherical-actuator-magnet manipulator (SAMM), to be used as an end-effector of a robot manipulator for robot-assisted magnetic manipulation. The SAMM uses three omniwheels to enable holonomic control of a spherical magnet's heading and enable the magnet's rotation axis to be set arbitrarily. The SAMM performs closed-loop control of its dipole using field measurements obtained from Hall-effect sensors. Prior work in robotic magnetic manipulation was limited by robot-manipulator joint limitations and singularities. We demonstrated that augmenting a robot manipulator with our singularity-free SAMM end-effector substantially eliminates these prior difficulties.

\section{REFERENCES}

[1] B. J. Nelson, I. K. Kaliakatsos, and J. J. Abbott, "Microrobots for minimally invasive medicine," Апnи. Rev. Biomed. Eng., vol. 12, pp. $55-85,2010$.

[2] J. L. Toennies, G. Tortora, M. Simi, P. Valdastri, and R. J. Webster III, "Swallowable medical devices for diagnosis and surgery: the state of the art," J. Mech. Eng. Sci., vol. 224, no. 7, pp. 1397-1414, 2010.

[3] G. Ciuti, P. Valdastri, A. Menciassi, and P. Dario, "Robotic magnetic steering and locomotion of capsule endoscope for diagnostic and surgical endoluminal procedures," Robotica, vol. 28, no. 2, pp. 199$207,2010$.

[4] M. T. Hou, H. -M. Shen, G. -L. Jiang, C. -N. Lu, I -J. Hsu, and J. A. Yeh, "A rolling locomotion method for untethered magnetic microrobots," Appl. Phys. Lett., vol. 96, no. 024102, pp. 1-3, 2010.
[5] S. Yim and M. Sitti, "Design and rolling locomotion of a magnetically actuated soft capsule endoscope," IEEE Trans. Robot., vol. 28, no. 1, pp. 183-194, 2012.

[6] A. W. Mahoney and J. J. Abbott, "Managing magnetic force applied to a magnetic device by a rotating dipole field," Appl. Phys. Lett., vol. 99, no. 134103, pp. 1-3, 2011.

[7] J. -S. Lee, B. Kim, and Y. -S. Hong, "A flexible chain-based screw propeller for capsule endoscopes," Int. J. of Prec. Eng. and Manuf., vol. 10, no. 4, pp. 27-34, 2009.

[8] L. Zhang, J. J. Abbott, L. X. Dong, B. E. Kratochvil, D. Bell, and B. J. Nelson, "Artificial bacterial flagella: Fabrication and magnetic control," Appl. Phys. Lett., vol. 94, no. 064107, pp. 1-3, 2009.

[9] K. Ishiyama, K. I. Arai, M. Sendoh, and A. Yamazaki, "Spiral-type micro-machine for medical applications," J. of Micromechatronics, vol. 2 , no. 1, pp. 77-86, 2003.

[10] A. W. Mahoney and J. J. Abbott, "Generating rotating magnetic fields with a single permanent magnet for propulsion of untethered magnetic devices in a lumen," IEEE Trans. Robot., vol. 30, no. 2, pp. 411-420, 2014.

[11] G. T. Gillies, R. C. Ritter, W. C. Broaddus, M. S. Grady, M. A. Howard III, and R. G. McNeil, "Magnetic manipulation instrumentation for medical physics research," Rev. Sci. Instrum, vol. 65, no. 3, pp. 533$562,1994$.

[12] J. R. Clark, L. Leon, F. M. Warren, and J. J. Abbott, "Magnetic guidance of cochlear implants: Proof-of-concept and initial feasibility study," Journal of Medical Devices, vol. 6, no. 035002, pp. 1-8, 2012.

[13] A. W. Mahoney and J. J. Abbott, "5-DOF manipulation of an untethered magnetic device in fluid using a single permanent magnet," in Proceedings of Robotics: Science and Systems, 2014.

[14] M. Kumaga and T. Ochiai, "Development of a robot balanced on a ball: Application of passive motion to transport," in Proc. IEEE Int. Conf. Robotics and Automation, 2009, pp. 4106-4111.

[15] U. Nagarajan, G. Kantor, and R. Hollis, "The ballbot: An omnidirectional balancing mobile robot," Int. J. Robot. Res., vol. 33, no. 6, pp. 917-930, 2014.

[16] M. Kumaga and R. L. Hollis, "Development of a three-dimensional ball rotation sensing system using optical mouse sensors," in Proc. IEEE Int. Conf. Robotics and Automation, 2011, pp. 5038-5043.

[17] K. -S. Byun and J. -B. Song, "Design and construction of continuous alternate wheels for an omnidirectional mobile robot," Journal of Robotic Systems, vol. 20, no. 9, pp. 569-579, 2003. 